\title{
Paul Suetens (ed): Fundamentals of Medical Imaging (2nd edition)
}

\author{
Cambridge University Press, Cambridge, UK 2009, 253 pages, ISBN: 978-0-521-51915-1
}

\author{
Rossella Ferrara $\cdot$ Luigi Mansi
}

Published online: 16 December 2010

(C) Springer-Verlag 2010

The book Fundamentals of Medical Imaging is edited by Paul Suetens, Professor of Medical Imaging and Image Processing at the University Hospital of Leuven, also working with apical commitments at the Department of Electrical Engineering of KU Leuven in Belgium. The publication, in its second edition arriving 7 years after the first one, mainly derives from his course on medical imaging for graduate and final year undergraduate students with a background in physics, mathematics or engineering.

The great challenge has been to produce a text useful not only for specialists, but for a wider series of readers, including biomedical scientists and medical practitioners in diagnostic imaging. The project is based on an editorial main construction of being "readable" also by physicians, connected with separate paragraphs including mathematical proofs that can be skipped without hampering a fluent reading of the text.

The publication explains the applied mathematical and physical principles of medical imaging and image processing and is organized into 8 chapters in 253 pages with 300 illustrations in colour.

The first chapter provides an introduction to digital imaging, explaining the physical and mathematical process. Over the next four chapters the most important diagnostic imaging modalities currently used are treated, including radiography, CT, MRI, nuclear medicine and ultrasounds. Each chapter is organized as follows:

R. Ferrara $\cdot$ L. Mansi $(\bowtie)$

Second University of Naples,

Naples, Italy

e-mail: luigi.mansi@unina2.it
1. Short historical introduction about the discovery of the treated imaging technique

2. Physical theory of signals and interaction with tissues

3. Imaging formation and reconstruction process

4. Debate on the quality of images

5. Different types of equipment used today

6. Examples of clinical applications

7. Description of the biological effects and safety issues

8. Some future expectations

The last two chapters deal with image analysis and visualization for diagnosis, including also tridimensional images, useful to better direct surgery and therapy. The book is further enriched by a description of future perspectives. An updated bibliography also includes, in a subdomain, suggestions for more specialized papers or for issues not treated in the book, but helpful for a deeper knowledge; finally, an appendix with exercises for better text comprehension is included. Answers, such as 3-D animations and other didactic material, can be obtained from an ancillary website (www.cambridge.org/suetens). This is one of the many implementations that have been added with respect to the first edition.

Coming back to the premise, i.e. to the editorial project of Paul Suetens, we can "certify" that the goal has been reached: recipients of the text Fundamentals of Medical Imaging are not only engineers, mathematicians and physicists, but also physicians and students involved in the field of diagnostic imaging.

This publication is particularly suggested for all diagnostic imaging laboratories, as a book to consult for finding valuable assistance in understanding the physical and mathematical principles of images as the basic premise to their clinical applications. 